\title{
BMJ Open The dose-response association of urinary metals with altered pulmonary function and risks of restrictive and obstructive lung diseases: a population-based study in China
}

Wei Feng, ${ }^{1}$ Xiji Huang, ${ }^{1}$ Ce Zhang, ${ }^{2}$ Chuanyao Liu, ${ }^{1}$ Xiuqing Cui, ${ }^{1}$ Yun Zhou, ${ }^{1}$ Huizhen Sun, ${ }^{1}$ Gaokun Qiu, ${ }^{1}$ Huan Guo, ${ }^{1}$ Meian He, ${ }^{1}$ Xiaomin Zhang, ${ }^{1}$ Jing Yuan, ${ }^{1}$ Weihong Chen, ${ }^{1}$ Tangchun $\mathrm{Wu}^{1}$

To cite: Feng W, Huang $X$, Zhang $C$, et al. The doseresponse association of urinary metals with altered pulmonary function and risks of restrictive and obstructive lung diseases: a populationbased study in China. BMJ Open 2015;5:e007643. doi:10.1136/bmjopen-2015007643

- Prepublication history and additional material is available. To view please visit the journal (http://dx.doi.org/ 10.1136/bmjopen-2015007643)

WF and XH contributed equally.

Received 12 January 2015 Revised 23 April 2015 Accepted 27 April 2015

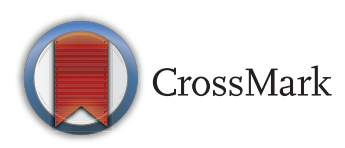

For numbered affiliations see end of article.

Correspondence to Professor Tangchun Wu; wut@mails.tjmu.edu.cn.

\section{ABSTRACT}

Objective: Reduced pulmonary function is an important predictor of environment-related pulmonary diseases; however, evidence of an association between exposures to various metals from all possible routes and altered pulmonary function is limited. We aimed to investigate the association of various metals in urine with pulmonary function, restrictive lung disease (RLD) and obstructive lung disease (OLD) risks in the general Chinese population.

Design: A cross-sectional investigation in the Wuhan cohort population.

Setting: A heavily polluted Chinese city.

Participants: A total of 2460 community-living Chinese adults from the Wuhan cohort were included in our analysis.

Main outcome measures: Spirometric parameters (FVC, forced vital capacity; $F E V_{1}$, forced expiratory volumes in $1 \mathrm{~s}$; FEV ${ }_{1} / F V C$ ratio), RLD and OLD.

Results: The dose-response associations of pulmonary function, and RLD and OLD, with 23 urinary metals were assessed using regression analysis after adjusting for potential confounders. The false discovery rate (FDR) method was used to correct for multiple hypothesis tests. Our results indicated that there were positive dose-response associations of urinary iron with $\mathrm{FEV}_{1}$ and $\mathrm{FEV}_{1} / \mathrm{FVC}$ ratio, vanadium with $\mathrm{FEV}_{1}$, and copper and selenium with $\mathrm{FEV}_{1} / \mathrm{FVC}$ ratio, while a negative dose-response association was observed between urinary lead and $\mathrm{FEV}_{1} / \mathrm{FVC}$ ratio (all $\mathrm{p}<0.05$ ). After additional adjusting for multiple comparisons, only iron was dose dependently related to $\mathrm{FEV}_{1} / \mathrm{FVC}$ ratio (FDR adjusted $p<0.05$ ). The dose-response association of iron and lead, with decreased and increased chronic obstructive pulmonary disease risk, respectively, was also observed (both $p<0.05$ ). Additionally, we found significant association of urinary zinc with RLD and interaction effects of smoking status with lead on $\mathrm{FEV}_{1} /$ FVC, and with cadmium on FVC and FEV 1 .

Conclusions: These results suggest that multiple urinary metals are associated with altered pulmonary function, and RLD and OLD prevalences.

\section{Strengths and limitations of this study}

- This is the first study using urinary data to investigate the associations of multiple metals with altered pulmonary function, and restrictive lung disease (RLD) and obstructive lung disease (OLD) risks, among the general Chinese population.

- We observed consistent evidence of doseresponse associations of iron and lead with altered pulmonary function and chronic obstructive pulmonary disease risk.

- Some metal concentrations in urine may not reflect real environmental exposure, and may therefore result in possible exposure misclassification.

- We estimated the levels of exposure to metals based on body burden data, which may not clarify the potential exposure pathway.

- Causal inferences cannot be made between metals and pulmonary function, and RLD and OLD risks, because of the cross-sectional design of the study.

\section{INTRODUCTION}

Reduced pulmonary function is an important predictor of cardiorespiratory morbidity and mortality, and chronic obstructive pulmonary disease (COPD) ${ }^{1-3}$ which was the third leading cause of death in China. ${ }^{4}$ There has also been a growing body of evidence suggesting that exposure to heavy metals such as arsenic, cadmium and lead, is associated with cardiopulmonary disease. ${ }^{5-8}$ However, evidence for the association of exposure to metals with altered pulmonary function, restrictive lung disease (RLD) and obstructive lung disease (OLD) risks among the general Chinese population, is largely unclear. Several epidemiological studies have focused on investigating the relationships of altered pulmonary function with exposure to arsenic from 
drinking water, cadmium and lead from cigarette smoking, ${ }^{9-12}$ iron and copper from dietary intake, ${ }^{13} 14$ and multiple metal irons in particulate matter. ${ }^{15}$ However, little is known of the relationship between exposure to various metals from all possible routes and altered pulmonary function. Given the fact that humans are exposed concomitantly to multiple metals through a variety of routes such as dietary intake, air particulate inhalation and drinking of water, it may be important for us to evaluate the health effects of various metals from all possible routes on pulmonary function.

Body burden monitoring of metals with theoretical advantages accounting for interindividual differences and all exposure routes is a useful tool to assess exposure to multiple metals. A recent study revealed that urinary arsenic was especially associated with impaired pulmonary function among 950 Bangladeshi patients with respiratory disease. ${ }^{16}$ Nevertheless, evidence from the general population with a large sample size is so far scarce. Moreover, although there is clear evidence that cigarette smoking is a major determinant for impaired pulmonary function and OLD risk, knowledge regarding the interaction effects of exposure to metals and smoking habits on pulmonary function was limited.

In the present study, we aimed to examine the associations of spirometric parameters, RLD, OLD and spirometrically defined COPD, with the urinary levels of 23 nutrient elements and heavy metals including aluminium, titanium, vanadium, chromium, manganese, iron, cobalt, nickel, copper, zinc, arsenic, selenium, rubidium, strontium, molybdenum, cadmium, tin, antimony, barium, tungsten, thallium, lead and uranium, among 2460 community-living Chinese adults. We also investigated the potential interaction of metals with smoking status on pulmonary function.

\section{METHODS}

\section{Study population}

We reported the baseline cross-sectional data from the Wuhan cohort study. Detailed information about the study designs and populations is provided in online supplementary material. The present analysis included 2460 participants from the cohort with available data of pulmonary function, urinary metals and other covariates.

\section{Definition of RLD and OLD}

We considered participants who responded that they currently had asthma, bronchitis and emphysema, and participants with self-reported and spirometry-defined COPD (forced expiratory volumes in $1 \mathrm{~s}, \mathrm{FEV}_{1} /$ forced vital capacity (FVC) ratio $<70 \%$ ) to have a current diagnosis of OLD. We defined other participants with $\mathrm{FEV}_{1} / \mathrm{FVC}$ ratio $\geq 70 \%$ and FVC $<80 \%$ predicted as patients with RLD.

\section{Determination of urinary metals and creatinine}

Metals in urine were analysed by inductively coupled plasma mass spectrometry. Duplicate analysis, spiked pooled sample, National Institute of Standards and Technology (NIST) Standard Reference Material (SRM) $2670 \mathrm{a}$ and 1640a, were used for quality control. A detailed description of the determination of urinary metal and quality control is included in online supplementary material. Urinary creatinine concentrations were measured by a fully automated clinical chemistry analyser.

\section{Pulmonary function testing}

Spirometry was performed using a digital spirometer interfaced to a computer (Chestgraph HI-101, CHEST MI, Inc, Tokyo, Japan) calibrated each morning according to the manufacturer's instruction. All spirometric examinations were performed with the participants in sitting position, wearing a nose clip and using a disposable mouthpiece. Each participants was asked to perform three satisfactory blows according to the recommendations of the American Thoracic Society. Values used in this analysis included the $\mathrm{FVC}, \mathrm{FEV}_{1}$ and the ratio of $\mathrm{FEV}_{1}$ to $\mathrm{FVC}$.

\section{Statistical analysis}

All analyses were conducted with the use of SPSS software. A $\mathrm{p}<0.05$ was considered statistically significant. Generalised linear regression models were used to assess the dose-response associations of $\mathrm{FVC}, \mathrm{FEV}_{1}, \mathrm{FEV}_{1} / \mathrm{FVC}$ ratio with 23 urinary metals as well as the interactions of metals with smoking status due to the skewed distribution of pulmonary function parameters. We also adopted logistic regression models to investigate the potential associations of RLD and OLD with 23 urinary metals. In order to robustly correct for multiple hypothesis tests, we used positive false discovery rate (FDR) method to adjust each $\mathrm{P}$ from 23 hypothesis tests. The FDR-adjusted $p$ value was calculated using a spreadsheet software provided by Pike. ${ }^{17} \mathrm{~A} p$ value was considered significant when the FDR-adjusted $p$ value of the test was less than 0.05. Finally, we examined the correlation between COPD and spirometry-related metals. Detailed illustrations of the statistical analysis are provided in online supplementary material.

\section{RESULTS}

\section{Descriptive statistics of participants}

The basic characteristics and pulmonary function parameters of the 2460 participants with and without lung diseases are summarised in table 1. The study population with a mean age of 52.3 years consisted of 1630 participants with normal pulmonary function, 602 individuals with RLD and 228 participants with OLD, which included 70 patients with COPD.

\section{Distribution of urinary metals}

The urinary excretion levels of 23 metals in the study sample are described in online supplementary table S1. Among 23 metals, most can be quantified in almost all samples except for tin and lead. 
Table 1 Basic characteristics and pulmonary function indexes of total population, participants with normal lung function, RLD and OLD in Wuhan City, China

\begin{tabular}{|c|c|c|c|c|}
\hline Variables & $\begin{array}{l}\text { Normal lung } \\
\text { function }(n=1630)\end{array}$ & RLD (n=602) & OLD $(n=228)$ & Total $(n=2460)$ \\
\hline Age, year & $50.6 \pm 12.7$ & $55.0 \pm 13.9$ & $59.8 \pm 12.5$ & $52.5 \pm 13.3$ \\
\hline \multicolumn{5}{|l|}{ Gender } \\
\hline Male & $544(33.3)$ & $248(41.2)$ & $90(39.5)$ & 882 (35.9) \\
\hline Female & $1086(66.6)$ & $354(58.8)$ & $138(60.5)$ & $1578(64.1)$ \\
\hline Height, m & $1.6 \pm 0.1$ & $1.6 \pm 0.1$ & $1.6 \pm 0.1$ & $1.6 \pm 0.1$ \\
\hline BMI, $\mathrm{kg} / \mathrm{m}^{2}$ & $24.1 \pm 3.3$ & $24.2 \pm 3.8$ & $24.2 \pm 3.5$ & $24.1 \pm 3.4$ \\
\hline \multicolumn{5}{|l|}{ Smoking status } \\
\hline Never & $1239(76.0)$ & $428(71.1)$ & $169(74.1)$ & $1836(74.6)$ \\
\hline Former & $78(4.8)$ & $44(7.3)$ & $21(9.2)$ & $143(5.8)$ \\
\hline Current & 313 (19.2) & $130(21.6)$ & $38(16.7)$ & $481(19.6)$ \\
\hline Pack year & $24.0 \pm 20.2$ & $32.0 \pm 28.0$ & $28.9 \pm 18.9$ & $26.7 \pm 22.8$ \\
\hline \multicolumn{5}{|l|}{ Alcohol status } \\
\hline Never & 1275 (78.2) & $458(76.1)$ & $186(81.6)$ & 1919 (78.0) \\
\hline Former & $54(3.3)$ & $26(4.3)$ & $12(5.3)$ & $92(3.7)$ \\
\hline Current & 301 (18.5) & $118(19.6)$ & $30(13.2)$ & 449 (18.3) \\
\hline \multicolumn{5}{|l|}{ Exercise intensity } \\
\hline Low activity & $944(57.9)$ & $324(53.8)$ & $116(50.9)$ & 1384 (56.3) \\
\hline Moderate activity & 401 (24.6) & $180(29.9)$ & 78 (34.2) & $659(26.8)$ \\
\hline High activity & $285(17.5)$ & $98(16.3)$ & $34(14.9)$ & $417(17.0)$ \\
\hline Serum haemoglobin*, g/L & $141.4 \pm 20.3$ & $141.2 \pm 18.4$ & $141.2 \pm 18.9$ & $141.3 \pm 19.7$ \\
\hline Urinary creatinine, $\mathrm{mmol} / \mathrm{L}$ & $13.6 \pm 7.8$ & $13.6 \pm 7.4$ & $13.2 \pm 7.7$ & $13.6 \pm 7.7$ \\
\hline FVC, L & $2.8 \pm 0.7$ & $2.0 \pm 0.6$ & $2.3 \pm 0.8$ & $2.6 \pm 0.8$ \\
\hline $\mathrm{FEV}_{1}, \mathrm{~L}$ & $2.4 \pm 0.6$ & $1.8 \pm 0.6$ & $1.9 \pm 0.7$ & $2.2 \pm 0.7$ \\
\hline $\mathrm{FEV}_{1} / \mathrm{FVC}$ ratio, $\%$ & $85.5 \pm 7.3$ & $91.2 \pm 8.3$ & $78.1 \pm 14.4$ & $86.2 \pm 9.1$ \\
\hline
\end{tabular}

\section{Pulmonary function and urinary metals}

The associations of metals with pulmonary function are presented in online supplementary table S2. After adjusting for age, gender, height, smoking status, pack year, alcohol status, body mass index (BMI), exercise and urinary creatinine, significant dose-response associations of urinary iron with elevated $\mathrm{FEV}_{1}$ and $\mathrm{FEV}_{1} /$ FVC ratio, urinary vanadium with increased $\mathrm{FEV}_{1}$, and urinary copper and selenium with increased $\mathrm{FEV}_{1} / \mathrm{FVC}$ ratio were seen, while urinary lead with decreased $\mathrm{FEV}_{1} /$ FVC ratio was observed (all $\mathrm{P}$ for trend $<0.05$ ). For example, relative to the referent (first quartile), the regression coefficients (95\% CIs) of $\mathrm{FEV}_{1}$ and $\mathrm{FEV}_{1} /$ FVC ratio in the fourth quintile of urinary iron were 0.085 (0.029 to 0.141$)$ and 1.796 (0.742 to 2.850), respectively; the regression coefficients (95\% CIs) of $\mathrm{FEV}_{1} / \mathrm{FVC}$ ratio in the third and fourth quartiles of urinary copper were 1.745 (0.697 to 2.793$)$ and 1.328 (0.187 to 2.468), respectively; and the regression coefficient (95\% CIs) in the third quartile of selenium was 1.348 (0.230 to 2.467). In contrast, compared with participants with urinary lead in the lowest quartile, decreased regression coefficients (95\% CIs) were estimated for $\mathrm{FEV}_{1} / \mathrm{FVC}$ ratio in participants with urinary lead in the fourth quartile $(\beta=-1.286 ; 95 \%$ CIs: -2.355 to -0.216). However, after additional adjusting for multiple hypothesis testing, only urinary iron was dosedependently associated with $\mathrm{FEV}_{1} / \mathrm{FVC}$.

\section{RLD and urinary metals}

We found that urinary zinc levels were dose-dependently associated with increased RLD risk after adjusting for potential cofounders $(\mathrm{p}<0.05)$. However, a significant association was not obtained after additional adjusting for multiple hypothesis testing. We also found that participants in the fourth quartile of urinary copper had a significant increase in RLD risk (ORs $=1.394$, 95\% CIs 1.018 to 1.909 ) compared with those in the first quartile, but there was a lack of dose-response trends. The results can be found in online supplementary table S3.

\section{OLD and urinary metals}

The results of association between OLD and 23 urinary metals are shown in online supplementary table S4. We found that urinary metals were not associated with OLD risk in multivariable models. However, results showed that there were dose-response associations of urinary iron and lead, with decreased and increased prevalence of COPD, respectively (both $\mathrm{p}<0.05$ ). As seen in table 2 , the ORs and 95\% CIs for the second through fourth quartiles relative to the referent of urinary iron were 0.431 (0.222 to 0.835$), 0.405$ (0.210 to 0.781$)$ and 0.335 
Table 2 The dose-response relationships of metals with COPD risk among Wuhan community residents, China

\begin{tabular}{|c|c|c|c|c|c|}
\hline \multirow[b]{2}{*}{ Variables } & \multicolumn{4}{|c|}{ Quartiles of urinary metals (units: $\mu \mathrm{g} / \mathrm{L}$ ) } & \multirow[b]{2}{*}{ p Value } \\
\hline & Q1 (lowest) & Q2 & Q3 & Q4 (highest) & \\
\hline Vanadium & $<0.336$ & $0.336-0.491$ & $0.492-0.748$ & $>0.748$ & \\
\hline $\mathrm{n}$ (case/control) & $23 / 597$ & $18 / 598$ & $15 / 598$ & $14 / 597$ & \\
\hline COPD & 1.000 (reference) & $0.815(0.418,1.589)$ & $0.736(0.365,1.487)$ & $0.780(0.374,1.628)$ & 0.455 \\
\hline Iron & $<44.864$ & $44.864-77.108$ & $77.109-142.849$ & $>142.849$ & \\
\hline $\mathrm{n}$ (case/control) & $30 / 597$ & $14 / 598$ & $15 / 598$ & $11 / 597$ & \\
\hline COPD & 1.000 (reference) & $0.431(0.222,0.835)$ & $0.405(0.210,0.781)$ & $0.335(0.161,0.698)$ & 0.002 \\
\hline Copper & $<5.199$ & $5.199-7.554$ & $7.555-11.210$ & $>11.210$ & \\
\hline $\mathrm{n}$ (case/control) & $26 / 598$ & $14 / 597$ & $9 / 598$ & $21 / 597$ & \\
\hline COPD & 1.000 (reference) & $0.550(0.273,1.111)$ & $0.331(0.145,0.753)$ & $0.735(0.363,1.488)$ & 0.291 \\
\hline Selenium & $<4.489$ & $4.489-7.639$ & $7.640-12.512$ & $>12.512$ & \\
\hline $\mathrm{n}$ (case/control) & $24 / 597$ & $16 / 599$ & $13 / 597$ & $17 / 597$ & \\
\hline COPD & 1.000 (reference) & $0.695(0.344,1.405)$ & $0.618(0.282,1.351)$ & $0.841(0.373,1.896)$ & 0.650 \\
\hline Lead & $<2.062$ & $2.062-3.164$ & $3.165-4.548$ & $>4.548$ & \\
\hline $\mathrm{n}$ (case/control) & $10 / 597$ & $23 / 598$ & $16 / 598$ & $21 / 597$ & \\
\hline COPD & 1.000 (reference) & $2.735(1.241,6.028)$ & $2.033(0.852,4.850)$ & $3.002(1.269,7.101)$ & 0.048 \\
\hline
\end{tabular}

(0.161 to 0.698$)$, respectively. At the same time, the ORs and $95 \%$ CIs for the second and fourth quartiles relative to the referent of urinary lead were 2.735 (1.241 to 6.028 ) and 3.002 (1.269 to 7.101), respectively. However, we did not find any dose-response association of vanadium, copper or selenium with COPD risk.

\section{Interaction of urinary metals and smoking status}

Subsequently, we investigated the difference in urinary levels of 23 metals among participants with different smoking status by multivariate analysis of covariance after adjusting for age, gender, BMI, alcohol status, exercise and urinary creatinine, and found that urinary nickel, zinc, cadmium, barium and lead levels were significantly higher in current smokers than those in neversmokers (FDR adjusted $\mathrm{p}<0.05$ or 0.001 , respectively, online supplementary table S5). We then examined the interaction effects of smoking status with vanadium, iron, copper, selenium, lead and cadmium on pulmonary function. Significant interactions of smoking status with lead on $\mathrm{FEV}_{1} / \mathrm{FVC}$ ratio, and cadmium on $\mathrm{FVC}$ and $\mathrm{FEV}_{1}$, were observed (all $\mathrm{P}$ for interaction $<0.05$; figure 1). Among current and former smokers but not among participants who had never smoked, urinary lead was significantly associated with decreased $\mathrm{FEV}_{1} / \mathrm{FVC}$ ratio. Also, urinary cadmium was strikingly correlated with FVC among current smokers rather than among former and never-smokers. However, we did not find significant interaction of vanadium, iron, copper and selenium with smoking habits on the three pulmonary function parameters (data not shown).

\section{DISCUSSION}

We observed consistent evidence of dose-response associations of iron and lead with altered pulmonary function and COPD risk, in linear and in logistic regression models. We also found that zinc was dosedependently associated with RLD but not pulmonary function and OLD risk, while vanadium and selenium were significantly and dose-dependently associated with increased pulmonary function but not RLD and OLD risks, based on the large Chinese population of community residents. Although we did not observe significant dose-response association between copper and RLD, participants in the fourth quartile of copper had a significant increase in RLD risk compared with those in the first quartile.

\section{Iron}

Iron is an essential metal for haemoglobin synthesis of erythrocytes, oxidation-reduction reactions and cellular proliferation, while excess iron accumulation causes organ dysfunction through the production of reactive oxygen species. ${ }^{18}$ Previous studies have suggested that there is positive association between serum iron and pulmonary function parameters among the US population and in male Japanese participants. ${ }^{14}{ }^{19}$ Consistently, we found in the population that significant associations existed between urinary iron and increased pulmonary function and decreased COPD risk. Our results suggested that current exposure levels of iron in our population did not contribute to airflow limitation but increased pulmonary function and COPD risk. Exposure to carbon monoxide from cigarette smoking and other routes can cause human disease, which was associated with a significant increase in haemoglobin levels. ${ }^{20}$ In the present study, we also examined the correlation between urinary iron and serum haemoglobin levels among the total population, and participants stratified by smoking status. The results can be seen in online supplementary table S6. We found that urinary iron was 
Figure 1 Interaction of lead and cadmium with smoking status on pulmonary function parameters.

All stratified analyses were adjusted for age, gender, height, alcohol status, body mass index, exercise and urinary creatinine. Data markers represent the estimated changes (error bars are $95 \mathrm{Cls}$ ) of pulmonary function indexes associated with per unit increasing natural log-transformed urinary lead and cadmium stratified by smoking status $\left(\mathrm{FEV}_{1}\right.$, forced expiratory volume in $1 \mathrm{~s}$; FVC, forced vital capacity).

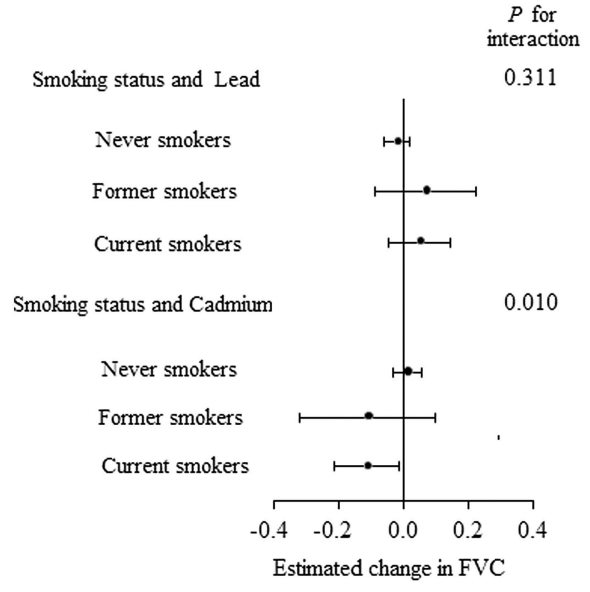

$P$ for
interaction

0.311

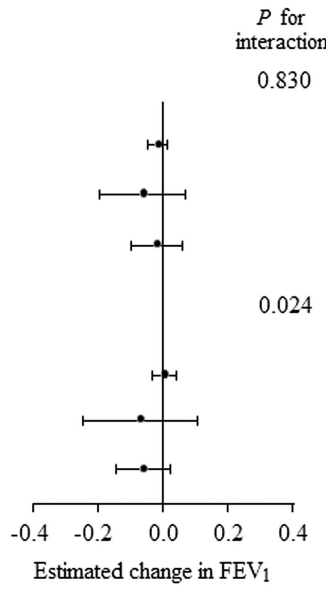

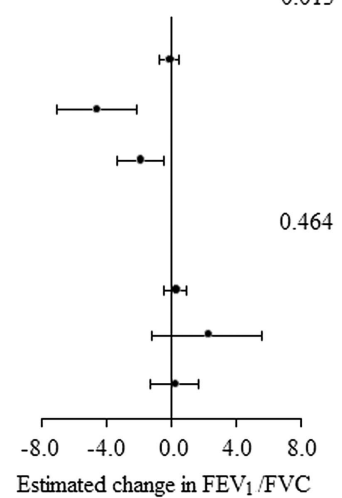

significantly associated with decreased haemoglobin levels among the total population and never-smokers, but not among smokers, although there were higher levels of haemoglobin in smokers than those in neversmokers (data not shown). It can be speculated that iron supplements may ameliorate damage induced by lowlevel exposure to carbon monoxide.

\section{Vanadium, copper and zinc}

Vanadium, copper and zinc have all been established as essential micronutrients and have numerous structural and biochemical roles in human health. ${ }^{21-23}$ Serum vanadium, copper and zinc were usually used as biomarkers to evaluate the deficiency of these elements in the human body. Imbalances (deficiency or excess) in the optimum levels of these elements may affect biological processes and can result in many diseases. In the present study, we also found that urinary vanadium was positively associated with $\mathrm{FEV}_{1}$. Nonetheless, there is currently little evidence suggesting that exposure to vanadium is associated with altered pulmonary function, despite a previous study indicating that occupational exposure to vanadium resulted in acute changes in pulmonary function. ${ }^{24}$ Moreover, Pearson et $a l^{13}$ found that serum copper was related to lower $\mathrm{FEV}_{1}$ among a UK general population. However, we found a positive doseresponse association between urinary copper and $\mathrm{FEV}_{1} /$ FVC among Chinese adults. We observed a dosedependent association between urinary zinc and RLD risk in the general population. A prior study suggested that sputum zinc was related to airway inflammation among participants with bronchiectasis. ${ }^{25}$ No previous study has investigated the association between the three metals in blood and urine, and (or) suggested which biomarkers may be better to reflect internal exposure to these metals. Meanwhile, we are unable to exclude the possibility of dependent measure error due to multiple metals measured in the same urine sample by the same assay. ${ }^{26}$ Thus, the present results and biological interpretations should be further investigated in future research.

\section{Selenium}

Selenium contributes to human antioxidant defences as an essential coenzyme in glutathione peroxides. Our results showed that urinary selenium was related to increased pulmonary function in the general Chinese population, which is consistent with previous studies suggesting the positive association of dietary and serum selenium with spirometric parameters in the general US and British population. ${ }^{13} 27$ It has been suggested that pulmonary function may be impaired by both free radical and oxidant exposure, while antioxidant intake is positively related to pulmonary function. ${ }^{28}$ Therefore, selenium intake may improve airflow obstruction by protecting against the injurious effects of free radicals or oxidants. $^{29}$

\section{Lead}

Lead is undoubtedly one of the oldest toxins. Several studies have examined the relationship of exposure to lead with cardiopulmonary disease. ${ }^{6} 30$ Only a recent study has shown that serum lead was associated with the increased risk of COPD in the National Health and Nutrition Examination Survey (NHANES) population and decreased $\mathrm{FEV}_{1}$ among these participants was stratified by smoking status. ${ }^{11}$ While the concentration of lead in blood was the most widely used indicator to monitor exposure, measurement of lead in urine has been used as a biomarker of internal dose, particularly for internal organic lead exposure. ${ }^{31} 32$ In the current study, although the COPD prevalence of $2.8 \%$ in our population was lower than that in the NHANES population $(12.4 \%)$, we still observed significant association of urinary lead with reduced $\mathrm{FEV}_{1} / \mathrm{FVC}$ ratio and increased COPD risk. The results were difficult to interpret, however, because the temporal sequence of causation could not be determined. Intriguingly, there were 3.5, 2.2 and 3.0 times higher geometric mean concentrations of urinary lead in our population as compared with those in the 2007-2008 NHANES, Belgian adult and Canada populations, respectively. ${ }^{33-35}$ Similarly, a recent study indicated that New Yorkers of Chinese 
origin have higher lead exposures than New Yorkers of Chinese descent not born in Mainland China. ${ }^{36}$ The reasons for the differences remain unclear and deserve further investigation. We speculated that the high levels of body lead burden in the Chinese population may at least in part be attributable to the high levels of airborne lead particles emitted mainly from heavy fuel oil, and therefore contribute to decreased pulmonary function in this population. ${ }^{15}$ Toxicological evidence on the mechanisms of lead-induced pulmonary function impairment is sparse. Some evidence has suggested that lead may involve the direct formation of reactive oxygen species, depletion of the cellular antioxidant pool and have other effects. ${ }^{37}$ Moreover, previous studies reported an association between oxidative stress and pulmonary function. ${ }^{28} 38$ Therefore, it has been postulated that oxidative stress may mediate lead-induced pulmonary function impairment.

\section{Effect modification}

Smoking habits are important determinants in reduced pulmonary function and developed COPD. ${ }^{39}$ Meanwhile, smokers have higher levels of toxic metals in their bodies. ${ }^{40}$ Our results suggested that higher urinary levels of cadmium in current smokers are associated with impaired pulmonary function, which is consistent with previous findings of negative associations of urinary cadmium with pulmonary function among smokers in the US population..$^{9-11}$ A previous study indicated that the adverse association of lead with pulmonary function was stronger in current smokers than in never-smokers. ${ }^{11}$ Similarly, our results revealed that there was an interaction between lead and smoking status, and a striking association with urinary lead was observed for decreased pulmonary function among these smokers but not among those without smoking.

\section{Study limitations}

First, a degree of measurement error may be present in this study due to multiple metals measured in the same urine sample by the same assay, coupled with using morning urine after overnight fasting instead of $24 \mathrm{~h}$ urine testing, which may be better for assessing the short-term variability in metal excretion and urine dilution. However, a $24 \mathrm{~h}$ urine collection, which is time consuming, cumbersome and improper, is less preferred than spot urine sample collection for biological monitoring of exposure in large population groups. Furthermore, the urinary level of metal adjusted for creatinine concentration in the present work may reduce the variability in the volume of urine and the metal content from void to void. Second, we did not evaluate the other confounders such as socioeconomic status and working environment. However, over-adjustments may also cause considerable bias. ${ }^{41}$ Third, some metal concentrations in urine may not reflect real environmental exposure, and therefore result in possible exposure misclassification. Further research is needed to detect the associations using other biological specimens such as blood and hair. Finally, we estimated the levels of exposure to metals based on body burden data, which may not clarify the potential exposure pathway. Further works are required to identify the possible routes of metals exposure, especially for lead exposure.

\section{CONCLUSION}

Our findings provide the first evidence suggesting a dose-response association between environmental exposure to metals and spirometric parameters, and RLD and COPD, in the general Chinese population. Our results also illustrate that there are interactions of heavy metal with smoking status on altered pulmonary function.

\section{Author affiliations}

${ }^{1}$ Key Laboratory of Environment and Health, Ministry of Education \& Ministry of Environmental Protection, and State Key Laboratory of Environmental Health (Incubation), School of Public Health, Tongji Medical College, Huazhong University of Science and Technology, Wuhan, Hubei, China ${ }^{2}$ Dongfeng Central Hospital, Dongfeng Motor Corporation and Hubei University of Medicine, Shiyan, Hubei, China

Contributors WF, XH, CZ, CL, XC, YZ and HS made substantial contributions to acquisition of the data, analysis and interpretation of the data. WF and $\mathrm{XH}$ drafted the article. GQ, HG, MH, XZ, JY, WC and TW revised the article critically for important intellectual content, and provided final approval of the submitted version. WC and TW are the guarantors of the data. TW had full access to all data in the study and takes responsibility for the integrity of the data and the accuracy of the data analysis. All authors approved the submitted version of the manuscript.

Funding This work was supported by funds from the National Key Basic Research and Development Program (973 project, grant number 2011CB503804, 2011CB503806 and 2011CB512102).

Competing interests None declared.

Patient consent Obtained.

Ethics approval The research protocol was approved by the Ethics and Human Subject Committee of Tongji Medical College, Huazhong University of Science and Technology.

Provenance and peer review Not commissioned; externally peer reviewed.

Data sharing statement No additional data are available.

Open Access This is an Open Access article distributed in accordance with the Creative Commons Attribution Non Commercial (CC BY-NC 4.0) license, which permits others to distribute, remix, adapt, build upon this work noncommercially, and license their derivative works on different terms, provided the original work is properly cited and the use is non-commercial. See: http:// creativecommons.org/licenses/by-nc/4.0/

\section{REFERENCES}

1. Drummond MB, Hansel NN, Connett JE, et al. Spirometric predictors of lung function decline and mortality in early chronic obstructive pulmonary disease. Am J Respir Crit Care Med 2012;185:1301-6.

2. Mannino DM, Buist AS, Petty TL, et al. Lung function and mortality in the United States: data from the First National Health and Nutrition Examination Survey follow up study. Thorax 2003;58:388-93.

3. Sin DD, Wu L, Man SF. The relationship between reduced lung function and cardiovascular mortality: a population-based study and a systematic review of the literature. Chest 2005;127:1952-9.

4. Yang G, Wang $Y$, Zeng $Y$, et al. Rapid health transition in China, 1990-2010: findings from the Global Burden of Disease Study 2010. Lancet 2013;381:1987-2015. 
5. Beveridge R, Pintos J, Parent ME, et al. Lung cancer risk associated with occupational exposure to nickel, chromium VI, and cadmium in two population-based case-control studies in Montreal. Am J Ind Med 2010;53:476-85.

6. Navas-Acien A, Guallar E, Silbergeld EK, et al. Lead exposure and cardiovascular disease-a systematic review. Environ Health Perspect 2007:115:472-82.

7. Menke A, Muntner P, Silbergeld EK, et al. Cadmium levels in urine and mortality among US adults. Environ Health Perspect 2009;117:190-6.

8. Chen CL, Hsu LI, Chiou HY, et al. Ingested arsenic, cigarette smoking, and lung cancer risk: a follow-up study in arseniasisendemic areas in Taiwan. JAMA 2004;292:2984-90.

9. Mannino DM, Holguin F, Greves HM, et al. Urinary cadmium levels predict lower lung function in current and former smokers: data from the Third National Health and Nutrition Examination Survey. Thorax 2004;59:194-8.

10. Lampe BJ, Park SK, Robins T, et al. Association between 24-hour urinary cadmium and pulmonary function among communityexposed men: the VA Normative Aging Study. Environ Health Perspect 2008;116:1226-30.

11. Rokadia H, Agarwal S. Serum heavy metals and obstructive lung disease: results from the National Health and Nutrition Examination Survey. Chest 2013;143:388-97.

12. von Ehrenstein OS, Mazumder DN, Yuan Y, et al. Decrements in lung function related to arsenic in drinking water in West Bengal, India. Am J Epidemiol 2005;162:533-41.

13. Pearson $\mathrm{P}$, Britton J, McKeever $\mathrm{T}$, et al. Lung function and blood levels of copper, selenium, vitamin $C$ and vitamin $E$ in the general population. Eur J Clin Nutr 2005;59:1043-8.

14. McKeever TM, Lewis SA, Smit HA, et al. A multivariate analysis of serum nutrient levels and lung function. Respir Res 2008;9:67.

15. Wu S, Deng F, Hao Y, et al. Chemical constituents of fine particulate air pollution and pulmonary function in healthy adults: the Healthy Volunteer Natural Relocation study. J Hazard Mater 2013;260:183-91.

16. Parvez $F$, Chen $Y$, Yunus $M$, et al. Arsenic exposure and impaired lung function. Findings from a large population-based prospective cohort study. Am J Respir Crit Care Med 2013;188:813-19.

17. Pike N. Using false discovery rates for multiple comparisons in ecology and evolution. Methods Ecol Evol 2011;2:278-82.

18. Kohgo $\mathrm{Y}$, Ikuta K, Ohtake T, et al. Body iron metabolism and pathophysiology of iron overload. Int J Hematol 2008;88:7-15.

19. Shibata $Y$, Inoue S, Igarashi A, et al. Elevated serum iron is a potent biomarker for spirometric resistance to cigarette smoke among Japanese males: the Takahata Study. PLoS ONE 2013;8:e74020.

20. Prockop LD, Chichkova RI. Carbon monoxide intoxication: an updated review. J Neurol Sci 2007;262:122-30.

21. Arredondo M, Núñez MT. Iron and copper metabolism. Mol Aspects Med 2005;26:313-27.

22. Goldwaser I, Gefel D, Gershonov E, et al. Insulin-like effects of vanadium: basic and clinical implications. J Inorg Biochem 2000;80:21-5.
23. Prasad AS, Bao B, Beck FW, et al. Antioxidant effect of zinc in humans. Free Radic Biol Med 2004;37:1182-90.

24. Lees RE. Changes in lung function after exposure to vanadium compounds in fuel oil ash. Br J Ind Med 1980;37:253-6.

25. Gray RD, Duncan A, Noble D, et al. Sputum trace metals are biomarkers of inflammatory and suppurative lung disease. Chest 2010;137:635-41.

26. Zeger SL, Thomas D, Dominici F, et al. Exposure measurement error in time-series studies of air pollution: concepts and consequences. Environ Health Perspect 2000;108:419-26.

27. Hu G, Cassano PA. Antioxidant nutrients and pulmonary function: the Third National Health and Nutrition Examination Survey (NHANES III). Am J Epidemiol 2000;151:975-81.

28. Schünemann HJ, Muti P, Freudenheim JL, et al. Oxidative stress and lung function. Am J Epidemiol 1997;146:939-48.

29. Cassano PA, Guertin KA, Kristal AR, et al. A randomized controlled trial of vitamin $E$ and seleniumon rate of decline in lung function. Respir Res 2015;16:35.

30. Jones SR, Atkin P, Holroyd C, et al. Lung cancer mortality at a UK tin smelter. Occup Med (Lond) 2007;57:238-45

31. Barbosa F Jr, Tanus-Santos JE, Gerlach RF, et al. A critical review of biomarkers used for monitoring human exposure to lead: advantages, limitations, and future needs. Environ Health Perspect 2005;113:1669-74.

32. Sanders T, Liu Y, Buchner V, et al. Neurotoxic effects and biomarkers of lead exposure: a review. Rev Environ Health 2009;24:15-45.

33. Authority of the Minister of Health. Report on human biomonitoring of environmental chemicals in Canada: results of the Canadian Health Measures Survey Cycle 1 (2007-2009). 2010.

34. CDC. Fourth national report on human exposure to environmental chemicals, updated tables, February 2012.

35. Hoet $P$, Jacquerye $C$, Deumer $G$, et al. Reference values and upper reference limits for 26 trace elements in the urine of adults living in Belgium. Clin Chem Lab Med 2013;51:839-49.

36. Muennig $P$, Wang $Y$, Jakubowski $A$. The health of immigrants to New York city from mainland China: evidence from the New York Health Examination and Nutrition Survey. J Immigr Refug Stud 2012;10:131-7.

37. Jomova K, Valko M. Advances in metal-induced oxidative stress and human disease. Toxicology 2011;283:65-87.

38. Ochs-Balcom HM, Grant BJ, Muti P, et al. Antioxidants, oxidative stress, and pulmonary function in individuals diagnosed with asthma or COPD. Eur J Clin Nutr 2006;60:991-9.

39. Anthonisen NR, Connett JE, Murray RP. Smoking and lung function of Lung Health Study participants after 11 years. Am J Respir Crit Care Med 2002;166:675-9.

40. Navas-Acien A, Selvin E, Sharrett AR, et al. Lead, cadmium, smoking, and increased risk of peripheral arterial disease. Circulation 2004;109:3196-201.

41. Greenland S. Modeling and variable selection in epidemiologic analysis. Am J Public Health 1989;79:340-9. 